\title{
A Multi-Objective Method for Short-Term Load Forecasting in European Countries
}

\author{
Mauro Tucci, Emanuele Crisostomi, Member, IEEE, Giuseppe Giunta, and Marco Raugi
}

\begin{abstract}
In this paper we present a novel method for daily short-term load forecasting, belonging to the class of similar shape algorithms. In the proposed method, a number of parameters are optimally tuned via a multi-objective strategy that minimises the error and the variance of the error, with the objective of providing a final forecast that is at the same time accurate and reliable. We extensively compare our algorithm with other state-of-the-art methods. In particular, we apply our approach upon publicly available data and show that the same algorithm accurately forecasts the load of countries characterized by different size, different weather conditions, and generally different electrical load profiles, in an unsupervised manner.
\end{abstract}

Index Terms-Short-term load forecasting, multi-objective optimisation, similar shape algorithms.

\section{NOMENCLATURE}

The following symbols are used throughout the paper. In particular we use bold letters to indicate vectors and matrices. In addition to the following symbols, we shall further use notation $\mathbf{V}^{(0)}$ to refer to vector $\mathbf{V}$ after removing its mean value.

\begin{tabular}{|c|c|}
\hline $\mathbf{L}_{N}$ & Load profile of the most recent $N$ days; \\
\hline $\mathbf{L}_{1}$ & Load profile of the most recent day; \\
\hline $\mathbf{H}_{N}$ & $\begin{array}{l}\text { An available historical load profile of } N \text { con- } \\
\text { secutive days; }\end{array}$ \\
\hline $\mathbf{B}_{N, j}$ & $\begin{array}{l}\text { The } j^{\prime} \text { th historical "best profile" similar to } \\
\mathbf{S}_{N} \text {; }\end{array}$ \\
\hline $\mathbf{B}_{1, j}$ & $\begin{array}{l}\text { The last day of the } j^{\prime} \text { th "best profile", also } \\
\text { denoted as the } j^{\prime} \text { th "best day"; }\end{array}$ \\
\hline $\mathbf{B}^{+1}(j)$ & $\begin{array}{l}\text { The historical value of the load in the day } \\
\text { after } \mathbf{B}(1, j) \text {; }\end{array}$ \\
\hline$d_{j}$ & Distance between $\mathbf{B}_{N, j}^{(0)}$ and $\mathbf{H}_{N}^{(0)}$ \\
\hline$s_{j}$ & Similarity between $\mathbf{B}_{N, j}^{(0)}$ and $\mathbf{H}_{N}^{(0)}$; \\
\hline$\hat{\mathbf{L}}_{1}$ & Reconstruction of $\mathbf{L}_{1}$ using the "best days"; \\
\hline$\hat{\mathbf{L}}$ & 24-hour ahead prediction; \\
\hline$N$ & $\begin{array}{l}\text { Number of the last available days. This pa- } \\
\text { rameter is obtained through an optimisation } \\
\text { procedure; }\end{array}$ \\
\hline M & $\begin{array}{l}\text { Number of best days to be considered. This } \\
\text { parameter is obtained through an optimisation } \\
\text { procedure; }\end{array}$ \\
\hline$\lambda$ & $\begin{array}{l}\text { Width factor of the Gaussian similarity Ker- } \\
\text { nel. This parameter is obtained through an } \\
\text { optimisation procedure; }\end{array}$ \\
\hline
\end{tabular}

M. Tucci, E. Crisostomi and M. Raugi are with the Department of Energy, Systems, Territory and Constructions Engineering, University of Pisa, Italy. Italy.
$N_{c a l} \quad$ Length of a calendar sequence. This parameter is obtained through an optimisation procedure;

W Diagonal matrix of weights $\left\{w_{1}, w_{2}, \ldots, w_{N}\right\}$ used to recombine the best days. This parameter is obtained through an optimisation procedure;

$\sigma \quad$ Standard deviation in the equation of the Gaussian similarity kernel;

$\alpha \quad$ Multiplicative scaling factor;

$\alpha^{*} \quad$ Optimal multiplicative scaling factor;

p Set of parameters used for a forecast;

$N_{\text {pred }} \quad$ Number of days used to make a comparison with another method;

$\mathbf{L}_{\text {true }}(i) \quad i^{\prime}$ the element of a time-series of true hourly load values used in the validation/comparison analysis;

$\mathbf{L}_{\text {pred }}^{p}(i) \quad$ Prediction of the $i^{\prime}$ th element of the hourly time-series $\mathbf{L}_{\text {true }}$ with set of parameters $\mathbf{p}$ used in the validation/comparison analysis;

$S P L F \quad$ Similar Profiles Load Forecast (proposed algorithm);

\section{INTRODUCTION}

$\mathbf{E}$ LECTRICAL load forecasting is an established yet still very active research topic due to a number of reasons: (i) the increasing penetration level of power generation from renewable sources has increased the amount of non-dispatchable energy that is injected in the power grid. An accurate forecast of the energy demand would thus allow energy providers to plan in advance an optimal scheduling of conventional power plants (e.g., thermoelectric plants) to support power generation to meet the energy demand; (ii) the recent ongoing deregulation of the electricity market has increased the competitiveness among energy retails. An accurate prediction of the energy demand would empower energy stakeholders with an important information to operate in the energy markets; (iii) finally, a better knowledge of the expected load would allow the power grid to operate in a more efficient way that not only would decrease operating costs, but also decrease the amount of polluting emissions in the air. For instance, load forecast is required to identify the expected load peaks during the day, and possibly recommend the use of peak shaving initiatives such as Demand Response or Load Shifting. Obviously, reducing the peak of the load reduces the requirement of maintaining some power plants switched on to operate just for a few minutes during the day, with high costs and high emissions throughout the day. Note that increasing the efficiency of the 
current power grid is one key step towards the realization of a truly smarter grid. In this paper, we are interested in shortterm load forecast, where the load of the whole day after is predicted once the load of the current day is known. A massive amount of research has already addressed such a load forecasting problem, and a short overview of the current state of the art is given in the next section.

\section{A. State of the art and paper contribution}

The annual number of scientific papers on load forecasting has increased from around one hundred in 1995 to more than a thousand in recent years ${ }^{1}$. Accordingly, it is hard to make a thorough state of the art, and here we only mention some of the papers that are mostly related to our methodology.

Load forecasting in the 90s was mainly tackled using neural network algorithms and linear regression methods [1]. One of the main challenges in adopting such methods relies in the fact that regular working days, holidays and special holidays (i.e., holidays that fall on otherwise regular working days) are characterised by completely different load profiles, and as such, should be treated in a different way. Accordingly, the basic algorithms have been recently modified to take into account such non-linear properties of the load, as described in a recent survey paper on load forecasting methods [2]. Generally speaking, the overall forecast is thus performed in two steps: in the first step, the day to be predicted is categorized according to its belonging to a week-day, or a holiday, or a pre-holiday, or other; then, the next day is predicted using an algorithm specialized on that category. Such approaches give rise to some hybrid methods where two algorithms are mixed together to perform the two steps (e.g., a Self-Organizing Map is used for the first step, and Support Vector Machines (SVMs) for the second one), see for instance [3], [4]. SVMs have been recently used by many other researchers as well, see for instance [5], [6] and [7]. In some cases, some algorithms have even been developed for a single category of days, see for instance [8], [9] that are dedicated to predict holidays and working days only, respectively.

As an alternative, other authors have developed algorithms that are sometimes denoted as "similar day-based" or "similar shape". In this case, the methods search in an available database for historical days that are characterised by similar weather conditions and/or similar weekday index to that of the day to be predicted. Then the prediction is computed by appropriately combining several similar days loads [10]. Such algorithms are particularly attractive because the belonging to a given cluster of data is intrinsically contained in the search for similar days (i.e., days similar to a holiday are usually automatically holidays). Many papers have been written exploiting this kind of methods; see for instance [11], where recurrent neural networks have been used to improve the similar days prediction; [12], where the focus in on very short-term load forecasts (one-to-six-hour-ahead); the already

${ }^{1}$ http://www.scopus.com mentioned [10], which also uses wavelet neural networks; and [13], which adopts a functional time-series methodology.

All the previous methods appear to have at least one of the following three limitations:

- they might only work for a subset of days (i.e., load forecast is performed only for a given class of days, e.g., working days);

- simulation results are given for a small window of time (e.g., a couple of months);

- experiments are conducted on a single set of data, which might make the reader wonder whether the proposed methodology depends on the specific data-set, or can be actually adopted to predict the load in other countries as well.

Following the previous discussion, the contributions of this paper are:

- We also propose a forecast algorithm within the class of similar shape algorithms. Differently from other papers, our algorithm automatically finds the similar profiles in the available data-base, and automatically works for all days of a year (i.e., working days, special holidays, ...). Our algorithm takes advantage of a number of parameters, whose optimal values are found according to a multi-objective optimisation problem that both aims to minimise the prediction error, and also the variance of the error. The rationale for this is to obtain an algorithm that is accurate (i.e., small error) and reliable (i.e., consistently small error) at the same time. Multi-objective algorithms are more rare to find in the load forecasting literature, see for instance [14], for an example for very-short-term forecasting (where the prediction horizon ranges from 5 to 30 minutes). In the context of load forecasting, it is also rare to find similarly automatic, totally unsupervised forecasting algorithms;

- Our forecasting algorithm outperforms, or performs similarly, other forecasting algorithms that use some further information (e.g., meteorological data). Also, our algorithms perform very well in completely different datasets, automatically tuning the optimisation parameters. More specifically, we test our algorithms on the Italian electrical load data, and compare our forecasts with those performed by the two main Italian forecast providers. Then we compare the Italian results with those obtained with other European countries that present different characteristics in terms of size, industrial load, weather conditions and electrical energy usages, and show that our algorithm, without any change, provides similar results in such different data-sets as well. Such a comparison on different data-sets is usually missing in the literature, and very few examples can be found (see for instance [15] where a meta-learning system was developed and cross-validated on different countries). Finally, we also compare the load forecasts of our algorithm with those obtained within the global energy forecasting competition in 2012. More details on the employed data-set are given 
in the next paragraph.

\section{B. Comparison and Performance}

The proposed algorithm has been extensively validated and tested on publicly available data, and performance results are illustrated in detail in Section IV. In particular, we perform three sets of tests.

At a national level: we first test the algorithm in the Italian national case, both on the data regarding the day-ahead market available in the $\mathrm{GME}^{2}$ website (which corresponds to the expected/actual load consumption as computed from market exchanged volumes of electrical energy), and on the data provided by Terna, which is the main transmission system operator (TSO) in Italy (and thus, corresponds to the expected/actual load consumption as computed from power flows in the power grid). We compare our accuracy results with the forecasts provided by GME, with those provided by $\mathrm{Terna}^{3}$, and with those provided by a simple regression algorithm, similar in the spirit to the one proposed in [16]. We shall show that our algorithm outperforms GME, and the regression algorithm, and provides results that are similar to those by Terna. This is a good result, since Ternas forecasts further use some exogenous signals (e.g., weather forecasts, and the information related to special events, like the broadcast on TV of the football world cup, or any other event that might have an impact on the electrical load).

At a European level: we then apply our algorithm to the electrical load of three other European countries, namely, Germany, France and Belgium. Such countries have been selected because they are representative of different latitudes with respect to Italy, and of different electrical load characteristics. Namely, Germany is characterised by a high industrial load that is pretty much constant throughout the year; France is characterised by a high winter load, due to the fact that electrical energy is often used for heating as well (as an alternative to gas); Belgium is characterised by having a size quite different from that of the other countries. Data for the electrical load in European countries is available from ENTSO-E ${ }^{4}$ data, where ENTSO-E is the European Network of Transmission Systems Operators for Electricity. In this case, we compare the performance of our algorithm in the different countries and show that the accuracy of the results is similar in the different countries. In our opinion, the fact that our algorithm has been tested on publicly available data, and that it performs well in all the selected countries, are two important merits of this paper, and we believe that our results can be used as a benchmark for other researchers that wish to compare their own algorithms in a fair and rigorous manner.

Small aggregations of electrical load: we finally compare the load forecasts of our algorithm with those obtained within the global energy forecasting competition in 2012, described

\footnotetext{
${ }^{2}$ https://www.mercatoelettrico.org/en/Default.aspx

${ }^{3}$ http://www.Terna.it/Default.aspx?tabid=101

${ }^{4}$ https://www.entsoe.eu/data/data-portal/consumption/Pages/default.aspx
}

in [22]. In this case the load time series pertains hourly loads in $k W$ (and temperature data) for a US utility with 20 zones at both the zonal (20 series) and system (sum of the 20 zonal level series) levels. Different zones have different electricity consumption behaviors and range from a few hundred of $k W$ to about $60 \mathrm{MW}$ in zone 9, relative to an industrial customer load.

This paper is organized as follows: Section II describes the overall algorithm; Section III illustrates how the proposed method is tuned according to a multi-objective procedure; Section IV illustrates the results that we have obtained in the Italian case, in the other European countries, and in the US utility case. Finally, conclusions of our paper are provided in the last section.

\section{Algorithm DESCRIPTION}

In the following, we assume that a historical database of the hourly load time-series is available. Then, given the load time-series up to the hour 24 of one day, our objective is to predict the 24 hourly load values of the next day, using all the past available data. The overall algorithm, that will be denoted as SPLF (Similar Profiles Load Forecast) in the remainder of the paper, is described in the flow chart depicted in Fig. 1. The single steps are now described in more detail:

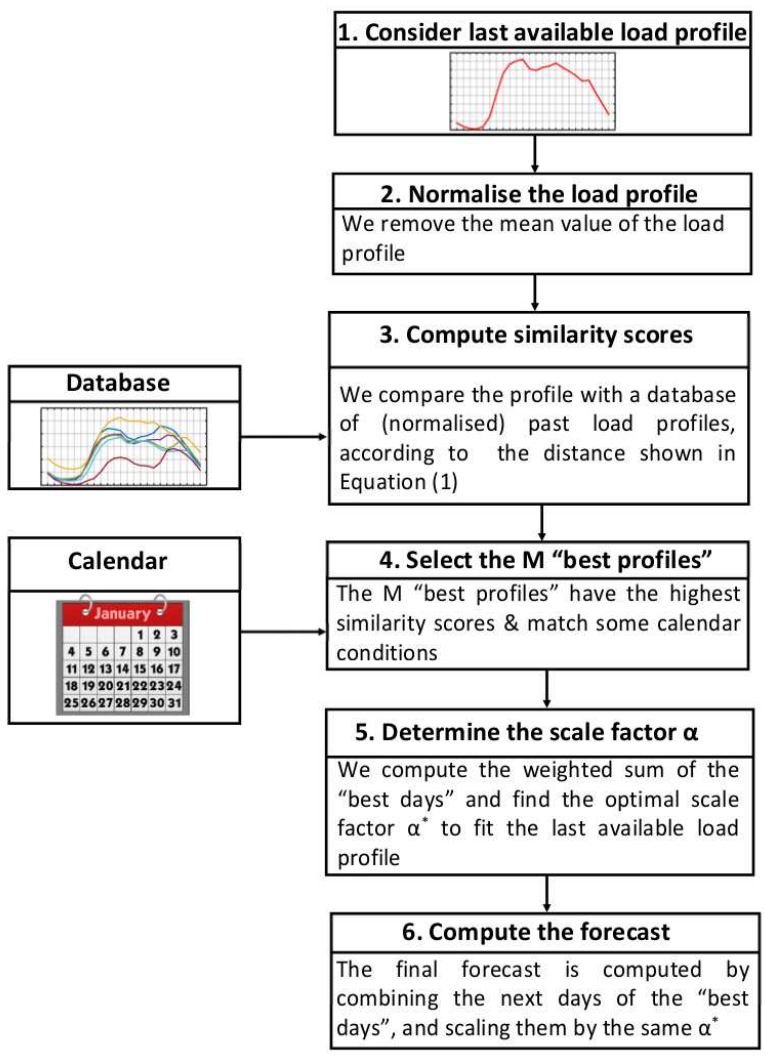

Fig. 1. Flow chart describing the steps of the SPLF algorithm.

1) We consider the most recent available $N$ days of the hourly load curve, and we denote it by $\mathbf{L}_{N}$. Similarly, $\mathbf{L}_{1}$ 
is the load curve of the last available day (e.g., today). The value of $N$ is a parameter of the algorithm, and its computation is explained in Section III.

2) We consider the zero-mean load curve $\mathbf{L}_{N}^{(0)}$ obtained from $\mathbf{L}_{N}$ after removing the mean value. We shall consider $\mathbf{L}_{N}^{(0)}$ as our "sample" load curve and we shall compare it with all the zero-mean load curves of the historical data-set. We denote by $\mathbf{H}_{N}$ a generic profile of $N$ days taken from the historical database, and by $\mathbf{H}_{N}^{(0)}$ its corresponding demeaned profile.

3) We determine the most similar profiles according to the weighted distance

$$
\left\|\mathbf{H}_{N}^{(0)}-\mathbf{L}_{N}^{(0)}\right\|_{\mathbf{W}^{2}}=\left\|\mathbf{W} \cdot\left(\mathbf{H}_{N}^{(0)}-\mathbf{L}_{N}^{(0)}\right)\right\|,
$$

where $\|\cdot\|$ is the Euclidean vector norm, and $\mathbf{W} \in$ $\mathbb{R}^{24 N \times 24 N}$ is a positive definite diagonal weight matrix, $\mathbf{W}=\operatorname{diag}\left\{w_{1}, w_{2}, \ldots, w_{24 N}\right\}, w_{k}>0$. The weight matrix is introduced to gain the flexibility to assign a different importance to different hours, and this is accomplished by optimising the parameters $w_{k}>0$. The distance (1) can be interpreted as a measure of dissimilarity between the last available (demeaned) load profile $\mathbf{L}_{N}^{(0)}$ and one historical (demeaned) load profile $\mathbf{H}_{N}^{(0)}$. Then we use the days after the most similar historical load profiles as a natural set of candidate forecasts for the load of tomorrow to be predicted.

4) However, even if some past load profile $\mathbf{H}_{N}^{(0)}$ is very close to $\mathbf{L}_{N}^{(0)}$, the day after $\mathbf{H}_{N}^{(0)}$ might not be a good candidate to predict the load of interest, e.g., the load of tomorrow. This happens if the calendar conditions in the past do not match the current calendar condition. Thus, we limit our attention only to the historical load profiles that satisfy some calendar conditions, as will be discussed in greater detail in Section II.C. In particular, we select the $M$ profiles that, among all those that satisfy the calendar conditions, have the smallest distance (1) from $\mathbf{L}_{N}^{(0)}$. We denote each of such best profiles (again, of $N$ days) as $\mathbf{B}_{N, j}^{(0)}$, where $j=1, \ldots, M$. We now convert the distance $d_{j}$ between $\mathbf{B}_{N, j}^{(0)}$ and $\mathbf{L}_{N}^{(0)}$, computed according to Equation (1), into a corresponding measure of similarity $s_{j}$ by using the Gaussian similarity kernel

$$
s_{j}=e^{-\frac{d_{j}^{2}}{\sigma^{2}}}, j=1, \ldots, M .
$$

The kernel width value in Equation (2) is defined as proportional to the smallest distance $d_{j}$, i.e., $\sigma=\lambda$. $\min _{j}\left\{d_{j}\right\}$, where $\lambda$ is a positive constant to be optimised. The choice of the Gaussian kernel as a function of the parameter $\lambda$ permits a large flexibility in the definition of the measures of similarity that will be used as weights to provide the load forecast.

5) In order to provide a final forecast, we first use the chosen best days to reconstruct the load of the last available 24 hours (i.e., the load of today). Accordingly,

$$
\hat{\mathbf{L}}_{1}=\sum_{j=1}^{M} s_{j} \mathbf{B}_{1, j},
$$

where $\mathbf{B}_{1, j}$ is the last day of the $j^{\prime}$ th best profile, and thus, we shall refer to it as the $j^{\prime}$ th "best day" in the remainder of this paper. We then determine the optimal scaling factor $\alpha^{*}$ that minimises the error distance between the true load and the reconstructed one as:

$$
\alpha^{*}=\underset{\alpha}{\operatorname{argmin}}\left\|\alpha \hat{\mathbf{L}}_{1}-\mathbf{L}_{1} \cdot\right\|
$$

6) Accordingly, the final forecast $\mathbf{L}^{*}$ is obtained by applying the same correction factor $\alpha^{*}$ to the weighted sum of the days after of the best days, i.e.,

$$
\mathbf{L}^{*}=\alpha^{*} \cdot \hat{\mathbf{L}}=\alpha^{*} \cdot \sum_{j=1}^{M} s_{j} \mathbf{B}^{+1}(j),
$$

where $\mathbf{B}^{+1}(j)$ is the 24 -component vector of the day after $\mathbf{B}(1, j)$.

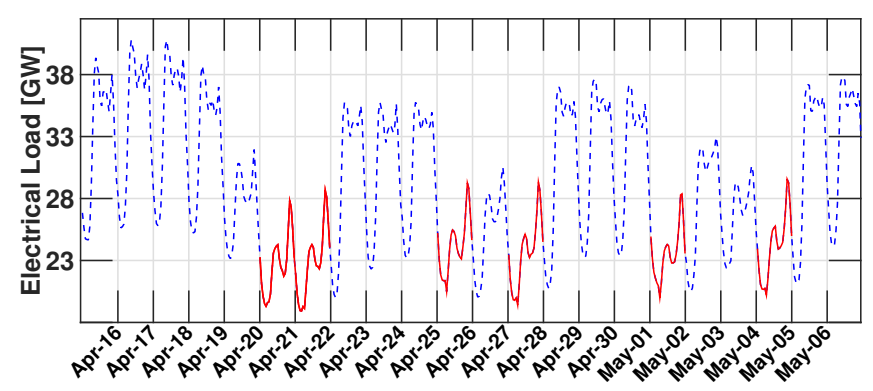

Fig. 2. Load with broken weekly periodicity, due to the occurrence of special holidays. The load during festive days is shown with a solid line.

\section{A. Calendar conditions}

The weekly periodicity of the load series (i.e., five consecutive week days with a high load, a Saturday with an intermediate load and a Sunday with a low load) is broken by the occurrence of special holidays during weekdays. An occurrence of this is shown in Fig. 2, which shows the Italian load in the Easter period in 2014. Due to the fact that also April 25th and May 1st are national holidays, the typical pattern does not appear anymore. As can be seen from the figure, it is of paramount importance to distinguish whether the load forecast involves a weekday or a holiday, since the load changes in a dramatic way. The importance of treating working days and holidays in a different way has been one of the main drivers of the recently proposed nonlinear forecasting algorithms as an alternative to traditional linear algorithms. To take this into account in our prediction, we divide the days of the week into three classes as follows:

1) Working Days: days from Monday to Friday, excluding special holidays.

2) Saturdays: all Saturdays excluding holidays.

3) Holidays: all Sundays and special holidays (Easter Monday, Christmas, New Year's Day, etc.).

The choice to cluster the daily load into the three previous classes follows simple intuitive analysis of the load (i.e., visual inspection), and has been justified in many papers in the literature, see for instance [17], [18], and [19] for the special case of European countries. Then, as anticipated in Section 


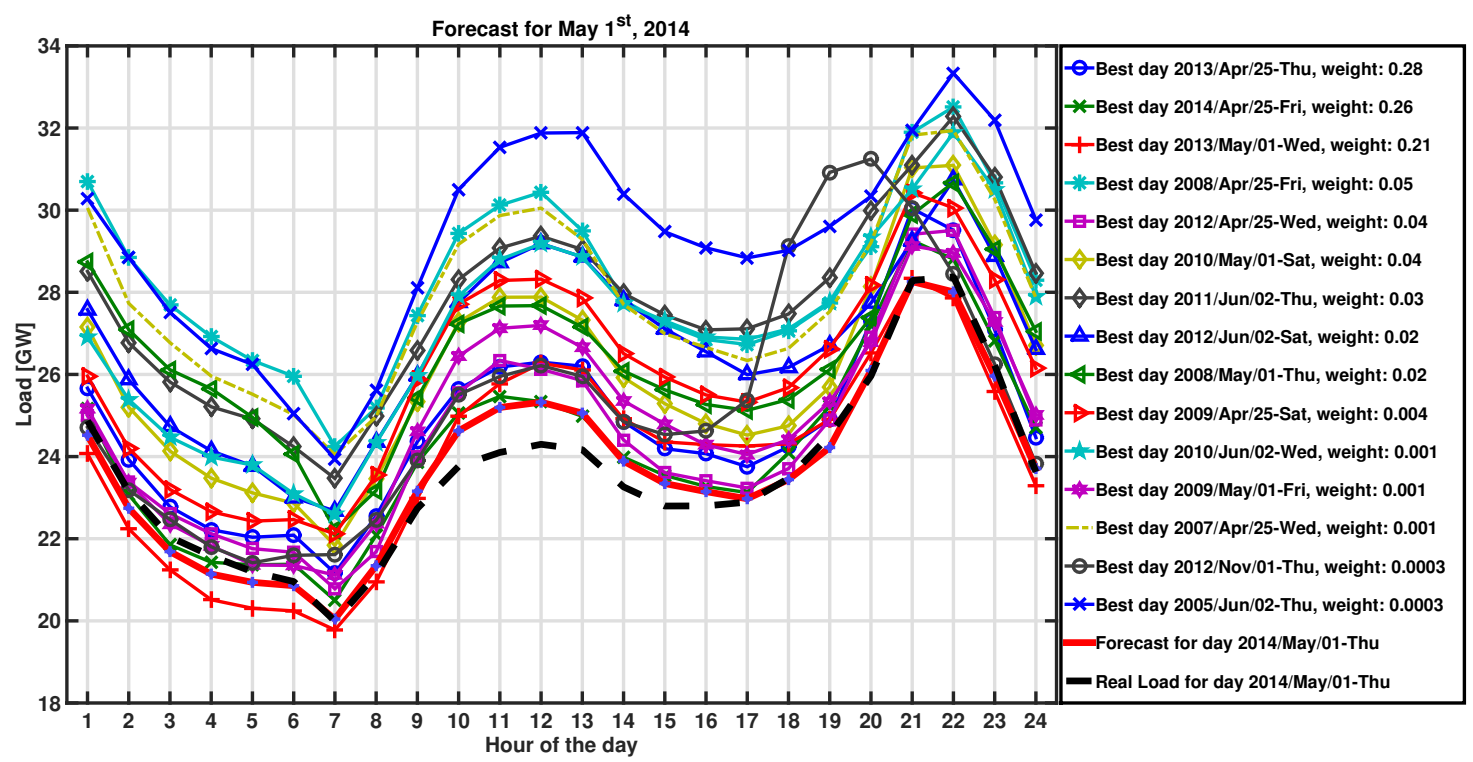

Fig. 3. Set of the best days and the final forecast for May 1 2014. As can be seen on the right, the algorithm autonomously and automatically identifies similar days (in terms of holidays and in terms of seasonality) as members of the set of best days.

II.B (step 4), we only retain the $M$ best days that also share the same sequence of calendar days. In particular, we require that the calendar should coincide for $N_{c a l}$ consecutive days (from the last available day), where $N_{c a l}$ is another parameter whose optimal value is obtained after an optimisation procedure as described in the next Section III. As an example of this, the prediction of the load for May 1st, 2014 is shown in Fig. 3. As can be seen, all the best days belong to similar calendar patterns in the previous years. The figure shows the final forecast $\mathbf{L}^{*}$, the pool of the load curves (the days after the best days) used for the weighted combination in (5) with the corresponding weights $s_{j}$, and also the actual load.

\section{PARAMETERS OPTIMISATION AND PERFORMANCE MEASURES}

The parameters of the SPLF algorithm to be optimised are:

1) $N$ : number of the last available days used to look for similar patterns in the past;

2) $M:$ number of the best days;

3) $\lambda$ : width factor of the Gaussian similarity kernel;

4) $\mathbf{W}=\operatorname{diag}\left\{w_{1}, w_{2}, \ldots, w_{N}\right\}$ : diagonal windowing matrix that defines the weighted distance in Equation 1;

5) $N_{\text {cal }}$ : the length of the calendar sequence.

For simplicity we have assumed that the diagonal elements of matrix $\mathbf{W}$ vary in a linear way so that only $w_{1}$ and $w_{N}$ need to be determined. Therefore, six parameters have to be optimised, and we shall denote the vector of parameters as $\mathbf{p} \in \mathbb{R}^{6}: \mathbf{p}=\left[N, M, \lambda, w_{1}, w_{N}, N_{\text {cal }}\right]^{T}$. We use SPLF to predict a number $N_{\text {pred }}$ of days in the past for which historical data are available. Then, we denote by $\mathbf{L}_{\text {pred }}^{p} \in \mathbb{R}^{24 N_{\text {pred }}}$ the column vector that contains all the performed 24 hours predictions obtained by using a vector of parameters $\mathbf{p}$, and by $\mathbf{L}_{\text {true }} \in \mathbb{R}^{24 N_{\text {pred }}}$ the column vector that contains the true load values during the corresponding period of $N_{\text {pred }}$ days. As a performance index, we use the Mean Absolute Percentage Error, $(M A P E)$ which can be defined as a function of $\mathbf{p}$ as:

$$
\operatorname{MAPE}(\mathbf{p})=\frac{100}{24 N_{\text {pred }}} \sum_{i=1}^{24 N_{\text {pred }}}\left|\frac{\mathbf{L}_{\text {pred }}^{p}(i)-\mathbf{L}_{\text {true }}(i)}{\mathbf{L}_{\text {true }}(i)}\right|
$$

$M A P E$ is the most used parameters in the load forecasting application field. Since MAPE only accounts for the accuracy purposes, here we further use the performance index relative to the Variance of the Absolute Percentage Error (VAPE), to further take into account the reliability of the forecast:

$$
\begin{aligned}
& V A P E(\mathbf{p})=\frac{1}{24 N_{\text {pred }}-1} . \\
& \cdot \sum_{i=1}^{24 N_{\text {pred }}}\left(100\left|\frac{\mathbf{L}_{\text {pred }}^{p}(i)-\mathbf{L}_{\text {true }}(i)}{\mathbf{L}_{\text {true }}(i)}\right|-M A P E(\mathbf{p})\right)^{2} .
\end{aligned}
$$

In order to determine the optimal set of solutions of the parameters $\mathbf{p}$, a multi-objective optimisation is performed by means of an evolutionary multi-objective optimisation algorithm, considering the objective functions 6 and 7 above. In particular, in this work we consider a variant of NSGA-II (Nondominated Sorting Genetic Algorithm), which is a controlled elitist genetic algorithm [20]. The NSGA-II approach is widely considered as a very efficient and good performing elitist multi-objective evolutionary algorithm. This is due, among others, to its reduced complexity thanks to the introduction of a fast non-dominated sorting approach, and to the use of a crowded comparison operator for diversity preservation. In this work we use the crowded comparison operator and calculate the distances in the objective function space (phenotype). We used a population size of 120 individuals, 6 variables and a Pareto fraction value of 0.35 (the solver will try to limit the number of individuals in the current population that are on the Pareto front to $35 \%$ of the population size). The algorithm stops when the maximum number of generations is reached, and we set this value to 1200 . It is worth to note that the optimisation step is in general quite time consuming (few hours), while in contrast the prediction step performed by 
SPLF is very fast (milliseconds to predict a whole year). Accordingly, the SPLF can be also used for very short-term forecasting (e.g., 15-minutes ahead), provided that the optimal parameters are recomputed at a slower time scale. The reason why we implement a multi-objective optimisation is that we search for load forecasts that are both accurate (i.e., small $M A P E)$ and reliable (i.e., small VAPE).

\section{NUMERICAL RESULTS}

In this section we show the performance of the SPLF algorithm. In the first part, we test the algorithm on the Italian load consumption data estimated from the electrical market and that estimated from electrical transmission data. We also compare our forecast method with other available forecasts. In the second part, we test the algorithm on a set of European countries of different latitudes, different size and different characteristics in terms of the electrical load. As a general trend of all the performed numerical simulations the choice of a yearly value of $N_{\text {pred }}$ is adequate to determine optimised parameters giving good performances of the SPLF on different years.

\section{A. Case study 1: Italian electrical load}

1) Pareto fronts of the multi-objective algorithm : We first consider the electrical load data-set provided by GME, for which historical data are available from 2005. We have found the optimal values of the parameters by predicting the days of the year 2012, taking into account both the MAPE and the VAPE indices, as illustrated in Section IV. Fig. 4 shows the Pareto front determined by the NSGA-II algorithm, representing 42 points that are equally optimal for year 2012 . The optimal values of year 2012 have then been used to predict the year 2013 (cross markers in Fig. 5), and are close to the Pareto front of that year (i.e., the optimal solution that we would have obtained if year 2013 was used to find the optimal parameters of the SPLF algorithm, shown with square markers). As already mentioned, the algorithm is quite robust because the values of the parameters that are optimal for one year remain close to optimality for the following year as well. This is particularly true if compared with a random set of parameters (chosen via Monte Carlo sampling from a uniform distribution), as the same Fig. 5 that shows with diamond markers the best solutions obtained via Monte Carlo sampling. This shows that an optimal choice of the parameters is required to get accurate results.

2) Comparison with other algorithms: In this paragraph, we compare SPLF with the forecasts developed by GME, Terna and those of a simple regression algorithm (denoted by EMP), inspired by the work of [16]. In the regression algorithm, the load of the next day is provided as a weighted combination of the load of the day before and the load of one week before (i.e., in the same day of the week of the day to be predicted). The weighting factors are optimally computed using the available data of past load profiles (using the MAPE index) and the algorithm is further enhanced by using calendar rules similar to those illustrated in Section II.A. Performance results are shown for each of the six areas in which the Italian load data is provided, and also for the national aggregated data. For the sake of clarity, in Table I, we only compare the different algorithms in terms of MAPE. Note that the results reported in Table I for SPLF and EMP have to be interpreted as "training results" in the year 2012, and as validation results for year 2013. In fact, load data were used to compute the optimal parameters of SPLF (and of EMP) in year 2012. On the other hand, results for year 2013 rely on parameters computed the year before, and accordingly can be interpreted as true validation results.

As can be seen from Table I, SPLF outperforms EMP in every single area, both for GME and for Terna data. Also, SPLF outperforms GME forecasts (which are also available from the GME website) in every area. At this regard, regional GME forecasts appear to make large errors, while the national forecast is much more accurate. The same trend can be noticed for Terna as well: although it is not known how GME and Terna make their forecasts, it could be that they are more interested, and consequently accurate, at a national level, and less accurate at a zone level. Still, both at a zone and at a national level, SPLF consistently improves GME forecasts as it is shown in Fig. 6, where the daily average $M A P E$ errors of the two forecasting algorithms are compared. Table I also allows us to compare SPLF with Terna forecasts. In this case, it appears that SPLF forecasts are more accurate at a regional level, while Terna forecasts are (slightly) more accurate at a national aggregate level.

Since SPLF's parameters had been optimised to minimise the $M A P E$, it remains an open question whether performance are weak with respect to other indices. For this purpose, Table II gives the results of the comparison with GME and Terna for what regards other indices as well. In Table II, RMSE is the Root Mean Square Error, MAE is the Mean Absolute Error, MAP is the Maximum Absolute Percentage error, MA is the Maximum Absolute error and MMAP is the Mean of daily Maximum Absolute Percentage error (see [14] and [8] examples of uses of such indices and their exact definition). Table II seems to suggest that the algorithm that outperforms the others according to one parameter, usually outperforms the others according to the other parameters as well. In Table II, SPLF MAPE refers to the case when SPLF's optimal parameters are chosen as those that minimise $M A P E$ in year 2012 (so, this corresponds to a single-objective optimisation of SPLF), SPLF VAPE refers to the case when SPLF's optimal parameters are chosen as those that minimise VAPE in year 2012 (so, this corresponds again to a single-objective optimisation of SPLF), and finally SPLF INT corresponds to a choice of parameters which is intermediate in the Pareto front of Fig. 4 (so this corresponds to a truly multi-objective optimisation of SPLF). So another result of Table II is that it is more convenient to train SPLF in a multi-objective fashion rather than in a single-objective fashion. As for many other methods reported in the literature, SPLF does not predict the (expected) uncertainties of daily forecasts. This would be an interesting aspect, and we are currently investigating the mechanism to provide an expected percentage of accuracy in 


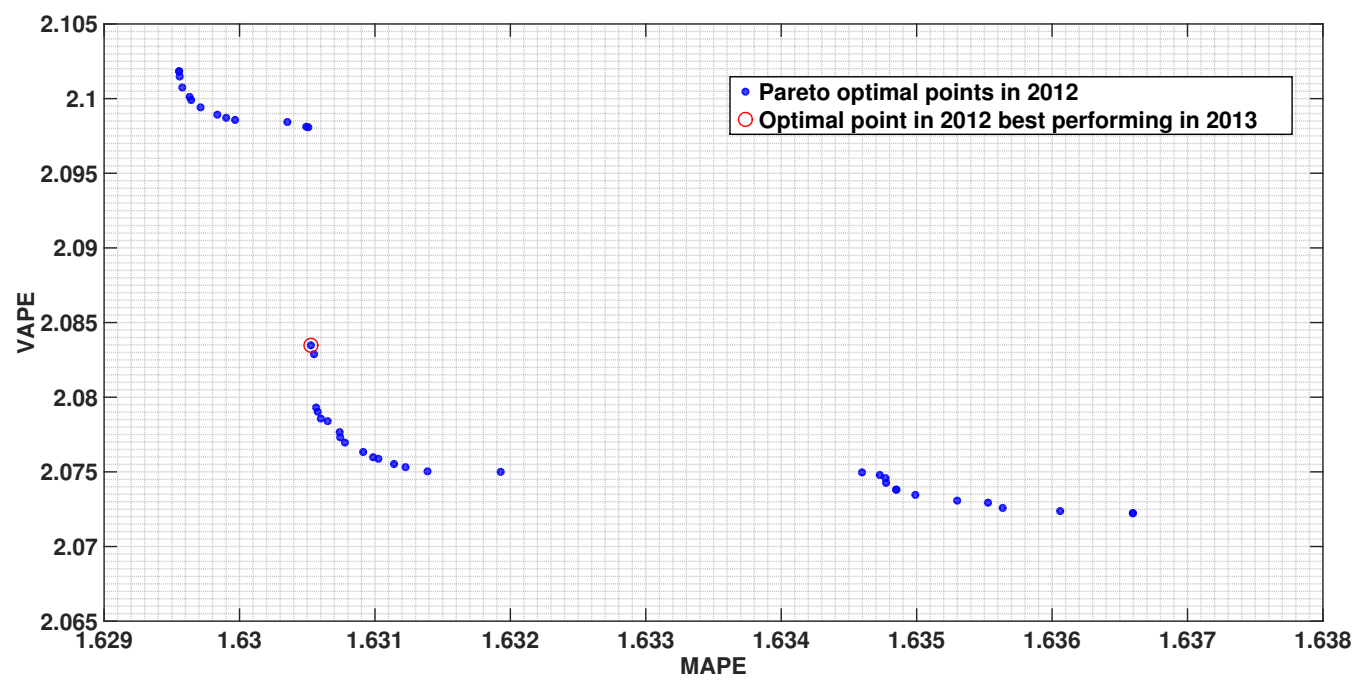

Fig. 4. Pareto optimal front in 2012. One particular solution of the Pareto optimal set in 2012, shown in a circle, is found to dominate all the other solutions in the following year.

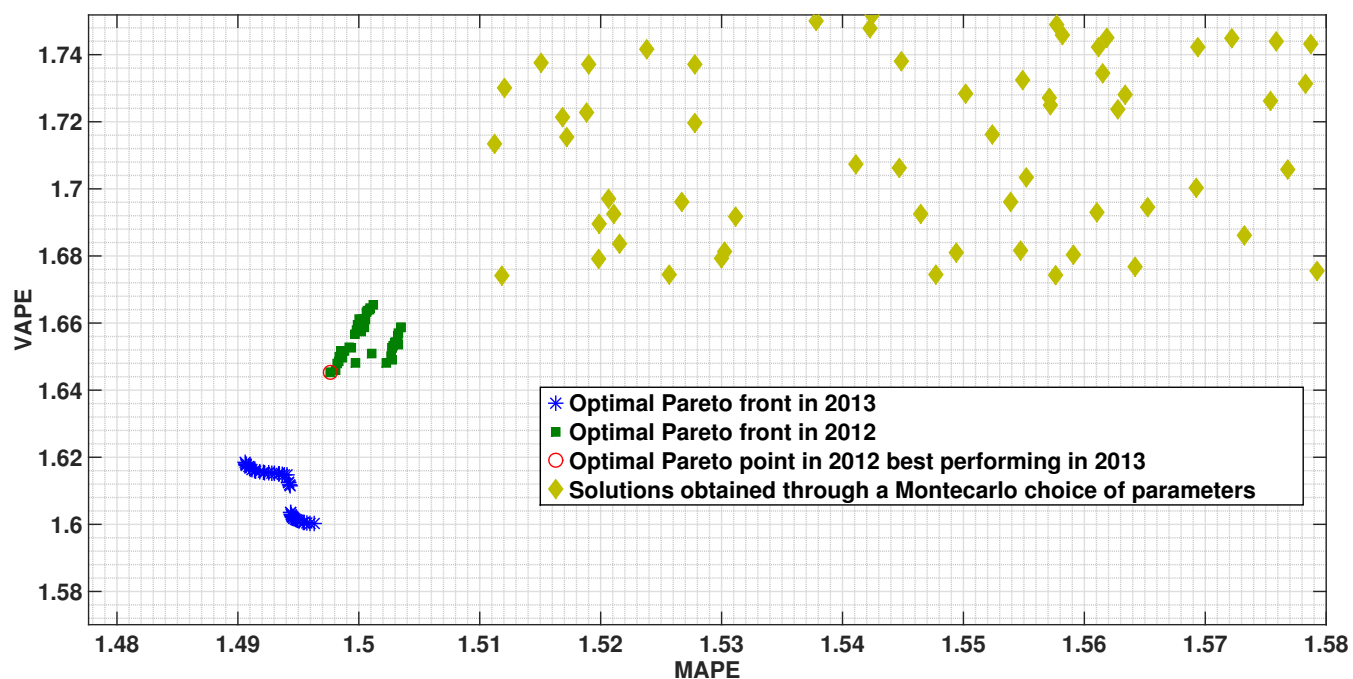

Fig. 5. Pareto optimal front in 2013, vs. solutions provided by the Pareto optimal front in 2012 and a Monte Carlo choice of the parameters. Although we only show the best solutions obtained via Monte Carlo choice of parameters, still they are far from the optimal set of solutions.

TABLE I

COMPARISON OF SPLF WITH EMP, GME AND TERNA FORECASTS. BEST RESUlTS ARE SHOWN IN BOLD.

\begin{tabular}{|c|c|c|c|c|c|c|c|c|c|c|c|c|}
\cline { 2 - 14 } \multicolumn{1}{c|}{} & \multicolumn{1}{c|}{2012 GME Data } & \multicolumn{2}{c|}{$\mathbf{2 0 1 3}$ GME Data } & \multicolumn{3}{c|}{ 2012 Terna Data } & \multicolumn{3}{c|}{ 2013 Terna Data } \\
\cline { 2 - 14 } \multicolumn{1}{c|}{} & SPLF & EMP & GME & SPLF & EMP & GME & SPLF & EMP & Terna & SPLF & EMP & Terna \\
\hline North & $\mathbf{2 . 1 4}$ & 3.64 & 5.49 & $\mathbf{1 . 8 8}$ & 3.63 & 4.23 & $\mathbf{2 . 2 3}$ & 4.42 & 2.35 & $\mathbf{2 . 3 4}$ & 4.48 & 2.50 \\
\hline Centre North & $\mathbf{2 . 2 7}$ & 3.09 & 5.59 & $\mathbf{2 . 9 5}$ & 3.47 & 13.44 & $\mathbf{3 . 6 7}$ & 4.78 & 4.04 & $\mathbf{3 . 9 9}$ & 5.52 & 4.75 \\
\hline Centre South & $\mathbf{1 . 8 6}$ & 2.46 & 4.86 & $\mathbf{2 . 1 7}$ & 2.68 & 6.70 & $\mathbf{2 . 9 0}$ & 3.87 & 3.48 & $\mathbf{3 . 0 5}$ & 3.75 & 3.83 \\
\hline South & $\mathbf{2 . 9 0}$ & 3.51 & 8.90 & $\mathbf{2 . 8 2}$ & 3.24 & 8.35 & $\mathbf{4 . 2 0}$ & 5.36 & 5.9 & $\mathbf{4 . 7 5}$ & 5.97 & 6.13 \\
\hline Sicily & $\mathbf{2 . 3 4}$ & 2.74 & 6.00 & $\mathbf{2 . 6 2}$ & 2.70 & 6.65 & $\mathbf{3 . 2 3}$ & 4.15 & 3.9 & $\mathbf{2 . 6 5}$ & 3.33 & 3.64 \\
\hline Sardinia & $\mathbf{2 . 5 8}$ & 3.63 & 16.00 & $\mathbf{3 . 3 9}$ & 3.73 & 20.26 & $\mathbf{2 . 8 5}$ & 3.52 & 3.54 & 4.01 & 4.68 & $\mathbf{3 . 8 7}$ \\
\hline Italy & $\mathbf{1 . 6 3}$ & 2.80 & 3.14 & $\mathbf{1 . 5 0}$ & 2.80 & 3.11 & 1.80 & 3.60 & $\mathbf{1 . 7 1}$ & 1.77 & 3.53 & $\mathbf{1 . 6 9}$ \\
\hline
\end{tabular}

the day ahead forecast. At present, we can only observe that we have an average MMAP of around 3.75 in a period of 4 years 2011-2014 for the GME and TERNA data set. This result shows a good performance on a statistically relevant data set, and we may conclude that the forecast is usually reliable, although the reliability of a single forecast is not fully quantified in advance.
Since SPLF and Terna provide very close forecasts in year 2013, next paragraph is dedicated to analyse whether such a difference is statistically relevant. In any case, as previously remarked, we still believe that it is already a good result that SPLF forecasts are comparable to those of Terna, since Terna uses some further information (e.g., weather 
TABLE II

COMPARISON IN 2013 DATA WITH GME AND TERNA WITH RESPECT TO OTHER INDICES AS WELL

\begin{tabular}{|c|c|c|c|c|c|c|c|c|}
\cline { 2 - 9 } \multicolumn{1}{c|}{} & \multicolumn{4}{c|}{ GME Data } & \multicolumn{3}{c}{ Terna Data } \\
\cline { 2 - 10 } \multicolumn{1}{c|}{} & SPLF MAPE & SPLF VAPE & SPLF INT & GME & SPLF MAPE & SPLF VAPE & SPLF INT & Terna \\
\hline RMSE & 639.13 & 644.59 & $\mathbf{6 3 8 . 8 8}$ & 1321.75 & 814.26 & 806.71 & 805.53 & $\mathbf{7 5 5 . 5 4}$ \\
\hline MAE & 484.96 & 489.75 & $\mathbf{4 8 2 . 3 9}$ & 1019.23 & 582.16 & 589.55 & 578.52 & $\mathbf{5 5 4 . 8 8}$ \\
\hline MAP & 17.08 & 16.15 & $\mathbf{1 4 . 9 4}$ & 17.07 & 18.12 & 15.97 & 15.95 & $\mathbf{1 5 . 0 2}$ \\
\hline MA & 3268.85 & 3242.45 & $\mathbf{3 1 9 7 . 3 1}$ & 5620.11 & 4830.91 & 4709.95 & 4713.78 & $\mathbf{4 4 1 6}$ \\
\hline MMAP & $\mathbf{3 . 4 6}$ & 3.57 & $\mathbf{3 . 4 6}$ & 6.4 & 4.11 & $\mathbf{4 . 0 7}$ & $\mathbf{4 . 0 7}$ & 4.09 \\
\hline
\end{tabular}

forecasts, knowledge of special events, ...) to elaborate their forecasts, which is not used in the SPLF case.

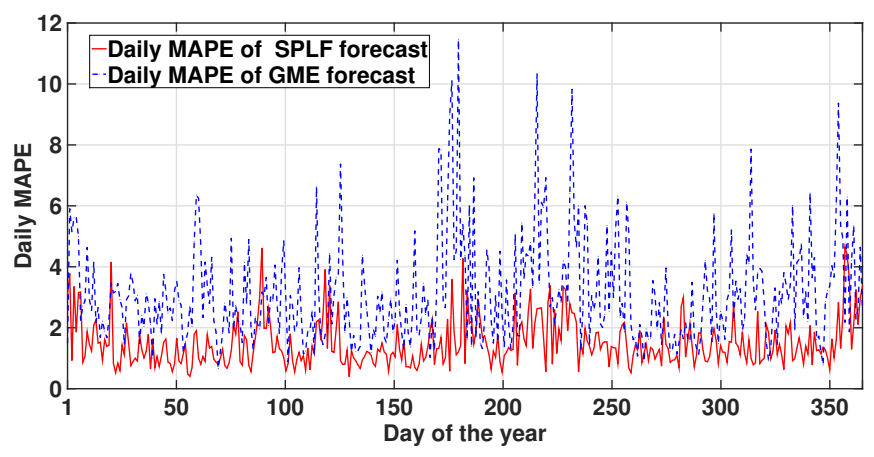

Fig. 6. Comparison of the average daily MAPE of SPLF and GME forecasts in year 2013 .

3) Statistical relevance: In the comparison, we use the set of parameters from the Pareto front in year 2012 with minimum MAPE, which corresponds to $\mathbf{p}=$ $[2,12,1.35,0.201,1.277,5]^{T}$. Note that such a solution is optimal for the Terna database (i.e., another data-set has a different optimal solution). Then we use the signed rank Wilcoxon test [21] to verify the statistical relevance of the $M A P E$ differences between the two forecasts, in different months of the year, and in different days of the year in Tables III and IV. A test result value of " 0 " denotes that

TABLE III

MONTHLY COMPARISON BETWEEN SPLF AND TERNA.

\begin{tabular}{|c||c|c|c||c|c|c|}
\hline \multicolumn{1}{|c||}{\multirow{2}{*}{$\begin{array}{c}\text { Terna } \\
\text { Italy }\end{array}$}} & \multicolumn{3}{c||}{$\mathbf{2 0 1 2}$ MAPE} & \multicolumn{3}{c|}{2013 MAPE } \\
\cline { 2 - 7 } Jan & SPLF & Terna & Test & SPLF & Terna & Test \\
\hline Feb & 1.53 & 2.21 & 0 & 1.56 & 1.50 & 0 \\
\hline Mar & 1.37 & 1.46 & 0 & 1.38 & 1.28 & 0 \\
\hline Apr & 2.10 & 1.88 & 0 & 1.75 & 1.58 & 0 \\
\hline May & 1.61 & 1.96 & 0 & 1.74 & 2.05 & 0 \\
\hline Jun & 2.36 & 1.83 & 0 & $\mathbf{1 . 6 1}$ & 2.03 & 0 \\
\hline Jul & 1.96 & 2.13 & 0 & 1.46 & 1.60 & 0 \\
\hline Aug & 2.20 & $\mathbf{1 . 6 3}$ & 1 & 2.45 & 2.25 & 0 \\
\hline Sep & $\mathbf{1 . 2 6}$ & 1.64 & 1 & 1.36 & 1.46 & 0 \\
\hline Oct & 1.22 & 1.27 & 0 & 1.36 & 1.40 & 0 \\
\hline Nov & 1.56 & 1.33 & 0 & 1.65 & 1.68 & 0 \\
\hline Dec & 2.16 & 1.71 & 0 & 2.50 & $\mathbf{1 . 7 3}$ & 1 \\
\hline Year & 1.80 & 1.71 & 0 & 1.77 & 1.69 & 0 \\
\hline
\end{tabular}

the MAPE differences are not statistically significant (i.e., the two methods are practically equivalent), while a value of " 1 " means that the $M A P E$ difference is statistically significant (the method with lower MAPE does outperform the other one). On
TABLE IV

PERFORMANCE IN WEEK DAYS USING AN OPTIMAL SOLUTION FOR TERNA 2012 .

\begin{tabular}{|c||c|c|c||c|c|c|}
\hline \multicolumn{1}{|c||}{\multirow{2}{*}{$\begin{array}{c}\text { Terna } \\
\text { Italy }\end{array}$}} & \multicolumn{3}{c||}{$\mathbf{2 0 1 2}$ MAPE} & \multicolumn{3}{c|}{ 2013 MAPE } \\
\cline { 2 - 7 } & SPLF & Terna & Test & SPLF & Terna & Test \\
\hline Mon & 2.17 & $\mathbf{1 . 5 5}$ & 1 & 2.03 & 1.95 & 0 \\
\hline Tue & 1.40 & 1.36 & 0 & 1.77 & 1.58 & 0 \\
\hline Wed & 1.73 & 1.81 & 0 & 1.56 & 1.50 & 0 \\
\hline Thu & 1.91 & 1.34 & 0 & 1.61 & $\mathbf{1 . 3 5}$ & 1 \\
\hline Fri & 1.59 & 1.47 & 0 & 1.70 & 1.66 & 0 \\
\hline Sat & $\mathbf{1 . 8 5}$ & 2.20 & 1 & 1.70 & 1.76 & 0 \\
\hline Sun & $\mathbf{1 . 7 2}$ & 2.16 & 1 & 1.80 & 1.88 & 0 \\
\hline Sp. Hol. & 2.76 & 2.23 & 0 & 2.35 & 2.39 & 0 \\
\hline
\end{tabular}

the basis of this test, SPLF is confirmed to provide forecasts that are statistically equivalent to those of Terna also for the national aggregated data. Also, it is possible to note that both approaches have a slightly worse performance during the special holidays (which on average correspond to 12 different days in Italy in one year). This confirms that such days are the most critical to predict.

\section{B. Case study 2: Comparison of the SPLF algorithm in different European countries}

One of the main benefits of the SPLF algorithm is that it can be directly applied to other countries as well. The only difference is that, obviously, the calendar rules have to be updated to take into account the specific national holidays of a given country. In this paper, we consider four different European countries, namely, Italy, France, Germany and Belgium, for which national aggregated data are publicly provided by ENTSO-E (see Fig. 7). There are some relevant differences among the electrical load in the four selected countries for a number of reasons:

- The countries belong to different latitudes, which gives rise to some different patterns. For instance, the weather is very hot in summer in Italy, and the electrical load is quite large due to air conditioning. Most commercial activities, offices, and some industries close for two weeks around August 15, when usually there are the hot days, and there is a dramatic decrease of the load. A similar pattern, though less evident, can be seen in the French case as well;

- The electrical load in France is particularly large in winter days. This is due to the fact that electrical energy is also used for heating, as an alternative to gas which is the conventional fuel in (most of) the other European countries; 

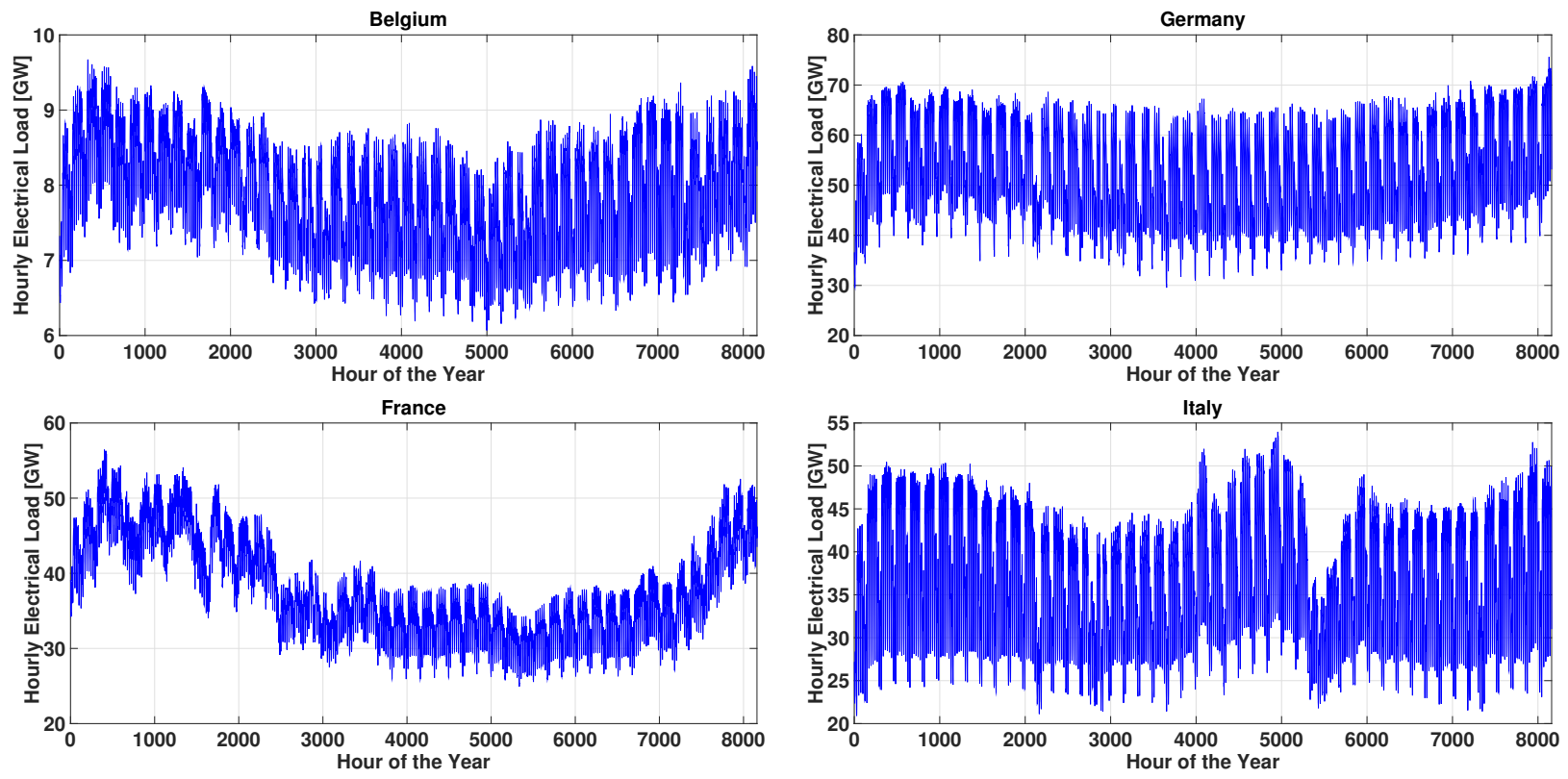

Fig. 7. Electrical load in 2013 in Belgium, Germany, France and Italy. Data are taken from the ENTSO-E database.

- The electrical load is practically constant throughout the year in Germany. This is mainly due to the fact that a significant component of the load is given by the industrial load that is in a large part not affected by seasonal patterns;

- Obviously, due to the smaller size, the electrical load in Belgium is smaller than that of the other countries.

The SPLF algorithm was applied to the four countries. The optimal parameters computed by the multi-objective optimisation procedure are reported in Table V. Interestingly, there are some relevant differences among the optimal parameters of different countries. Despite such differences, it is worthy to note that the performance of the algorithms in the different European countries (the average MAPE in year 2013) is similar, as shown in Tables VI and VII. Furthermore, the average accuracy

TABLE V

ALGORITHM PARAMETERS.

\begin{tabular}{|c||c|c|c|c|c|c|}
\hline \multicolumn{1}{|c||}{ ENTSO-E } & \multicolumn{6}{c|}{ Optimal Parameters for ENTSO-E 2012 } \\
\cline { 2 - 7 } Load & $N$ & $M$ & $\lambda$ & $w_{1}$ & $w_{n}$ & $N_{\text {cal }}$ \\
\hline Belgium & 2 & 10 & 1.28 & 0.27 & 1.45 & 3 \\
\hline Germany & 1 & 16 & 1.52 & 0.19 & 1.97 & 3 \\
\hline France & 1 & 8 & 1.97 & 0.25 & 1.17 & 2 \\
\hline Italy & 1 & 11 & 1.16 & 0.79 & 1.49 & 3 \\
\hline
\end{tabular}

of the obtained results is also similar to that reported in the literature by other methods on different sets of data, when also temperature data were considered, see for instance [10]. This evidences the robustness and the effectiveness of the multiobjective optimisation approach. Also, special holidays remain the most difficult to predict, as already remarked by other authors in the literature, as shown in Table VII. The accuracy of the prediction is shown in Fig. 8 for the critical period mentioned in Fig. 2. Fig. 9 provides a sensitivity analysis of the choice of parameters. For this purpose, we randomly perturbed the optimal parameters given in Table $\mathrm{V}$ in a range of $\pm 10 \%$
TABLE VI

MAPE RESULTS FOR ENTSO-E DATA IN 2013.

\begin{tabular}{|c||c|c|c|c|}
\hline \multicolumn{1}{|c|}{$\begin{array}{c}\text { ENTSO-E } \\
\text { Load }\end{array}$} & \multicolumn{4}{|c|}{ SPLF $\mathbf{M A P E}$} \\
\cline { 2 - 5 } Jan & Belgium & Germany & France & Italy \\
\hline Feb & 1.76 & 2.15 & 1.80 & 1.37 \\
\hline Mar & 2.09 & 1.60 & 2.15 & 1.23 \\
\hline Apr & 2.02 & 2.89 & 2.39 & 1.64 \\
\hline May & 1.88 & 2.08 & 2.48 & 1.94 \\
\hline Jun & 1.71 & 1.98 & 1.03 & 1.66 \\
\hline Jul & 1.76 & 1.32 & 0.95 & 1.74 \\
\hline Aug & 1.93 & 1.41 & 1.31 & 2.94 \\
\hline Sep & 1.55 & 1.39 & 1.06 & 1.67 \\
\hline Oct & 2.36 & 2.10 & 1.68 & 1.56 \\
\hline Nov & 1.98 & 1.88 & 1.78 & 2.11 \\
\hline Dec & 2.49 & 2.67 & 1.90 & 2.61 \\
\hline Year & $\mathbf{1 . 9 3}$ & $\mathbf{1 . 9 0}$ & $\mathbf{1 . 7 3}$ & $\mathbf{1 . 8 2}$ \\
\hline
\end{tabular}

TABLE VII

MAPE RESULTS FOR ENTSO-E DATA IN 2013.

\begin{tabular}{|c||c|c|c|c|}
\hline \multicolumn{1}{|c||}{$\begin{array}{c}\text { ENTSO-E } \\
\text { Load }\end{array}$} & \multicolumn{4}{c|}{ SPLF MAPE } \\
\cline { 2 - 5 } Mon & Belgium & Germany & France & Italy \\
\hline Tue & 2.24 & 2.05 & 1.87 & 2.37 \\
\hline Wed & 1.87 & 1.54 & 1.63 & 1.80 \\
\hline Thu & 1.65 & 1.61 & 1.46 & 1.47 \\
\hline Fri & 1.82 & 1.91 & 1.56 & 1.29 \\
\hline Sat & 1.77 & 2.08 & 1.84 & 1.75 \\
\hline Sun & 2.00 & 2.01 & 1.88 & 1.77 \\
\hline Sp. Hol. & 3.28 & 3.28 & 2.17 & 3.20 \\
\hline
\end{tabular}

for the real parameters, and \pm 1 for the natural parameters. It is interesting to notice that although optimal parameters were different from country to country, still forecasting results in all countries are mostly sensitive to the values of $N$ and $N_{c a l}$. In fact, as shown in Fig. 9, optimal results appear in small clouds of points that are separated by the values of such two important parameters. 

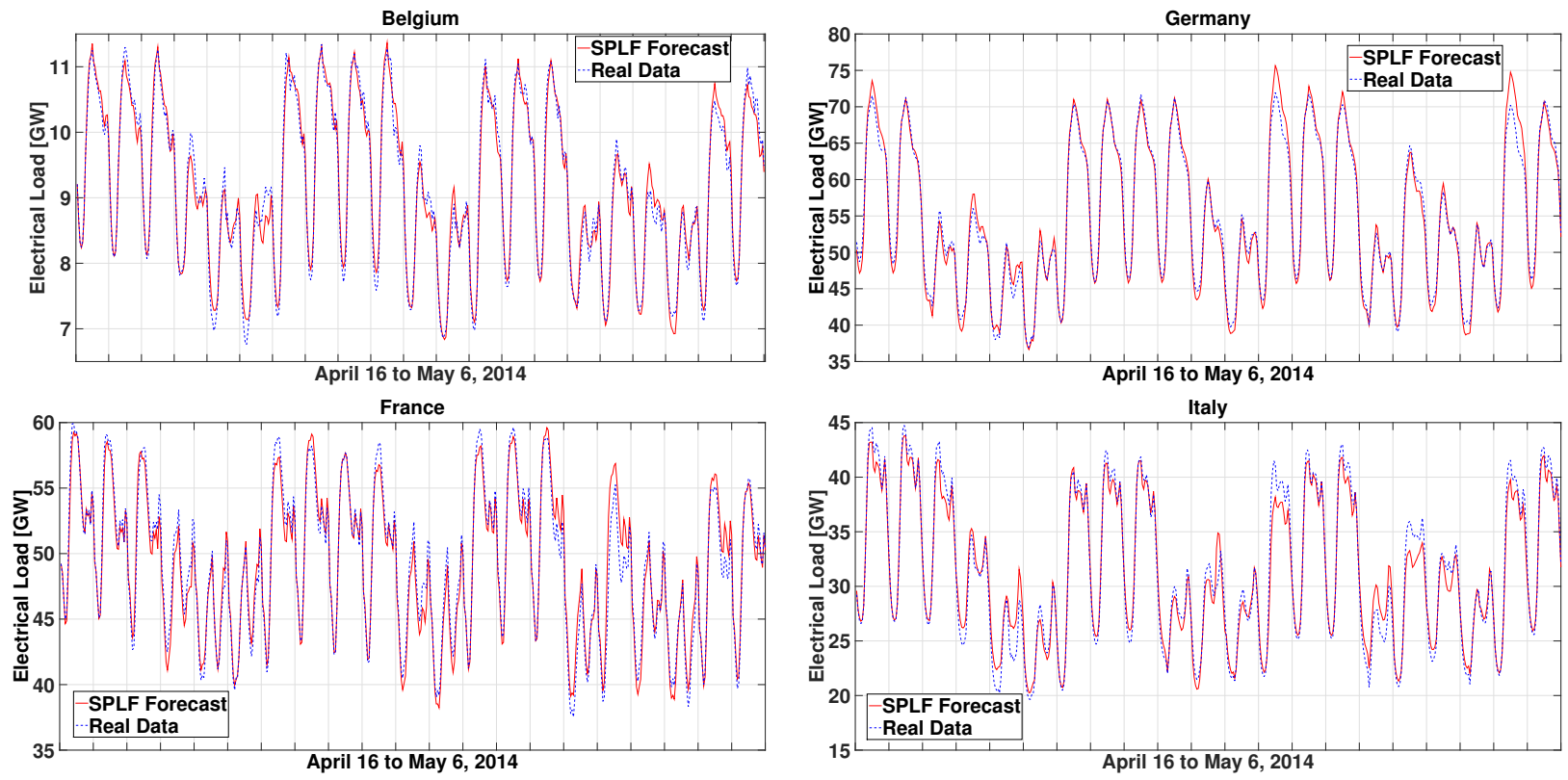

Fig. 8. SPLF forecasts in Belgium, Germany, France and Italy, in the critical period around Easter 2014 (i.e., with some special holidays). Data are taken from the ENTSO-E database.
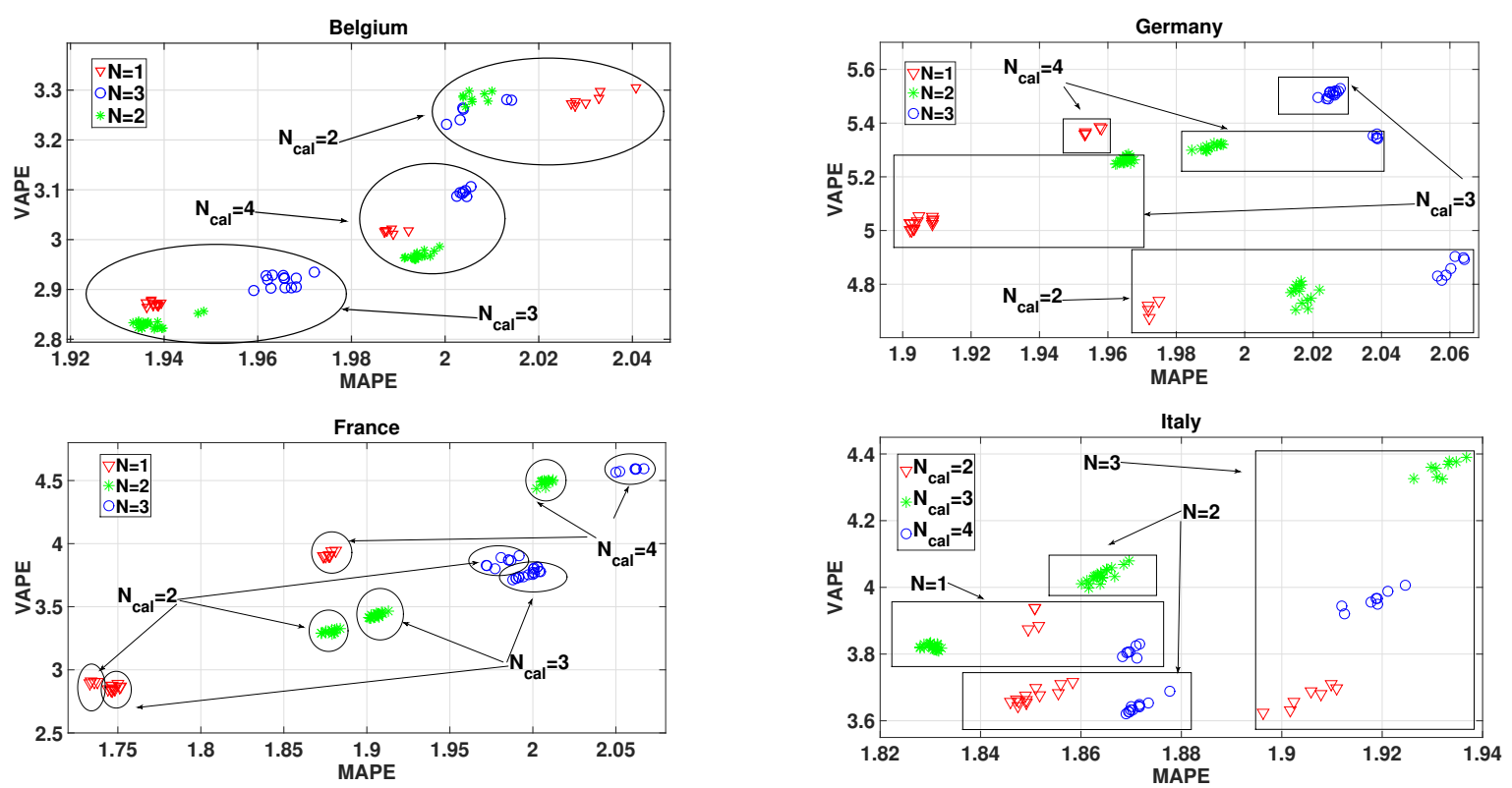

Fig. 9. Sensitivity analysis of the optimised parameters in the four considered countries. In all cases, $N$ and $N_{\text {cal }}$ appear to be the most sensitive parameters, as a wrong choice of such parameters might significantly affect the performance of the final forecast.

C. Case study 3: Application to the load time series described in [22]

The last case study refers to the global energy forecasting competition that took place in 2012, whose final results have been recently published in [22]. In such a competition, the participants were required to backcast and forecast hourly loads (in $\mathrm{kW}$ ) for a US utility for 20 different zones corresponding to different types of load (e.g., end-user loads, industrial loads), and in the sum of all the zones. The organisers of the competition provided a database of 4.5 years of hourly load and temperature data, from which they removed eight non-consecutive weeks of load data. The final task was to predict the hourly value of the load in the missing 8 weeks in the past (backcast) and in the week immediately after the available series. Note that temperature values were given for the 8 weeks in the past, and not in the week in the future. The load predictions were then compared according to a Weighted Root Mean Square Error (WRMSE), whose definition is provided in [22], together with more details about how the final score was computed.

In order to make a fair comparison with the other algorithms 
participating to the same competition, in this paper we use our SPLF algorithm only to predict the week in the future for which temperature data were not available and thus, unless predicted, could not be used by the other algorithms either. Note that the comparison is not truly fair for a number of reasons: (i) SPLF was designed to perform on a time-series corresponding to an aggregate load. This is not the case for the last case study. As a consequence, the load curves do not always exhibit the typical calendar profiles that have been investigated in this paper; (ii) the parameters of SPLF were computed according to a multi-objective cost function that takes into account MAPE and VAPE, and then we evaluated its performance according to a (slightly) different cost function (WRMSE); (iii) SPLF provide a 24-hour ahead prediction, and here it was also used in a recursive fashion to predict a whole week (by simply using the last 24-hour prediction as a true value of the load). Despite the previous differences, we still decided to provide the obtained results to evidence the robustness of the provided algorithm even when used in a slightly different framework.

Table VIII shows the results obtained by benchmark prediction, and the best 9 predictions of the competition. In this comparison, we also report in the last line the results obtained by SPLF. The table shows that SPLF outperforms all the other algorithms for what regards the prediction of the next 24 hours. On the other hand, its performance is not as competitive as the others for what regards the prediction of the whole week, but still the result is (slightly) better than the one obtained by the benchmark prediction.

TABLE VIII

RESULTS OF THE FORECASTING TASK OF THE COMPETITION

\begin{tabular}{|c||c|c|}
\hline \multicolumn{1}{|c|}{ Participant } & $\begin{array}{c}\text { 1-day ahead } \\
\text { WRMSE }\end{array}$ & $\begin{array}{c}\text { 1-week ahead } \\
\text { WRMSE }\end{array}$ \\
\hline Counting Lab & 72504 & 73900 \\
\hline James Lloyd & 59273 & 82346 \\
\hline Tololo (EDF) & 52136 & 82776 \\
\hline TinTin & 112410 & 86590 \\
\hline Quadrivio & 63186 & 81645 \\
\hline Chaotic Experiments & 50967 & 89783 \\
\hline Andrew L & 133005 & 106272 \\
\hline NHH & 121818 & 109850 \\
\hline The Jelly Team & 120752 & 101066 \\
\hline Tao's Vanilla Benchmark & 148352 & 123758 \\
\hline SPLF & 28084 & 119928 \\
\hline
\end{tabular}

\section{CONCLUSiON}

In this paper a novel unsupervised algorithm based on a "similar shape" approach for short-time forecast of the electrical load was presented. With respect to the many other existing methods for load forecasting, our paper presents two main contributions: (i) the algorithm is optimised to automatically provide a 24-hour ahead forecast that is at the same time accurate and reliable, thanks to the proposed multi-objective procedure for tuning some suitably introduced parameters of the algorithm; and (ii) the same algorithm can be applied in different test cases with similar results. For instance, it has been tested with similar good results to the load data of some different European countries. Thanks to such a second feature, the proposed forecasting method appears to be robust with respect to different load data characteristics, as further emphasised in the last test-case relative to a small aggregated load. Furthermore, the average accuracy (MAPE) of the obtained results is comparable to that obtained by other methods that also include temperature data.

\section{ACKNOWLEDGMENT}

The authors would like to thank the (anonymous) reviewers whose remarks contributed to improve the quality and the clarity of the final version of this article.

\section{REFERENCES}

[1] H. S. Hippert, C. E. Pedreira and R. C. Souza, Neural Networks for ShortTerm Load Forecasting: a Review and Evaluation, IEEE Transactions on Power Systems, vol. 16, no. 1, pp. 44-55, 2001.

[2] J. W. Taylor, L. M. de Menezes and P. E. McSharry, A comparison of univariate methods for forecasting electricity demand up to a day ahead, International Journal of Forecasting, vol. 22, pp. 1-16, 2006.

[3] T. Senjyu, P. Mandal, K. Uezato and T. Funabashi, Next day load curve forecasting using hybrid correction method, IEEE Transactions on Power Systems, vol. 20, no. 1, pp. 102-109, 2005.

[4] S. Fan and L. Chen, Short-term load forecasting based on an adaptive hybrid method, IEEE Transactions on Power Systems, vol. 21, no. 1, pp. 392-401, 2006.

[5] B.-J. Chen, M.W. Chang and C.J. Lin, Load forecasting using support vector machines: A study on EUNITE competition 2001, IEEE Transactions on Power Systems, vol. 19, no. 4, pp. 1821-1830, 2004.

[6] D. Niu, Y. Wang and D.D. Wu, Power load forecasting using support vector machine and ant colony optimization, Expert Systems with Applications, vol. 37, no. 3, pp. 2531-2539, 2010.

[7] E. Ceperic, V. Ceperic and A. Baric, A Strategy for Short-Term Load Forecasting by Support Vector Regression Machines, Expert Systems with Applications, vol. 28, no. 4, pp. 4356-4364, 2013.

[8] K.-B. Song, Y.-S. Baek, D.H. Hong and G. Jang, Short-term load forecasting for the holidays using fuzzy linear regression method, IEEE Transactions on Power Systems, vol. 20, no. 1, pp. 96-101, 2005.

[9] J. W. Taylor, Short-term Load Forecasting with Exponentially Weighted methods, IEEE Transactions on Power Systems, vol. 27, no. 1, pp. 458-464, 2012.

[10] Y. Chen, P. B. Luh, C. Guan, Y. Zhao, L. D. Michel, M. A. Coolbeth, P. B. Friedland and S. J. Rourke, Short-Term Load Forecasting: Similar Day-Based Wavelet Neural Networks, IEEE Transactions on Power Systems, vol. 25, no. 1, pp. 322-330, 2010.

[11] T. Senjyu, P. Mandal, K. Uezato and T. Funabashi, Next day load curve forecasting using recurrent neural network structure, IEE Proceedings on Generation, Transmission and Distribution, vol. 151, no. 3, pp. 388394, 2004.

[12] P. Mandal, T. Senjyu, N. Urasaki and T. Funabashi, A neural network based saveral-hour-ahead electric load forecasting using similar days approach, Elec. Power and Energy Systems, vol. 28, pp. 367-373, 2006.

[13] E. Paparoditis and T. Sapatinas, Short-term load forecasting: the similar shape functional time-series predictor, IEEE Transactions on Power Systems, vol. 28, no. 4, pp. 3818-3825, 2013.

[14] M. Alamaniotis, A. Ikonomopoulos and L. H. Tsoukalas, Evolutionary Multiobjective Optimization of Kernel-Based Very-Short-Term Load Forecasting, IEEE Transactions on Power Systems, vol. 27, no. 3, pp. 1477-1484, 2012

[15] M. Matijaš, J. A. K. Suykens and S. Krajcar, Load forecasting using a multivariate meta-learning system, Expert Systems with Applications, vol. 40, pp. 4427-4437, 2013.

[16] A.-H. Mohsenian-Rad and A. Leon-Garcia, Optimal residential load control with price prediction in real-time electricity pricing environments, IEEE Transactions on Smart Grid, vol. 1, no. 2, 2010.

[17] G. Chicco, R. Napoli and F. Piglione, Load pattern clustering for shortterm load forecasting of anomalous days, IEEE Power Technology Proceedings, vol. 2, Porto, 2001.

[18] L. Semeraro, E. Crisostomi, A. Franco, G. Giunta, A. Landi, M. Raugi and M. Tucci, Electrical Load Clustering: the Italian case, IEEE PES Conference on Innovative Smart Grid Technologies (ISGT) Europe, Istanbul, 2014. 
[19] A.K. Tanwar, E. Crisostomi, P. Ferraro, G. Giunta, M. Raugi and M. Tucci, Clustering analysis of the Electrical Load in European Countries, The 2nd International Workshop on Computational Energy Management in Smart Grids, @ International Joint Conference on Neural Networks (IJCNN), Killarney, Ireland, 2015.

[20] D. Kalyanmoy, Multi-objective optimization using evolutionary algorithms, John Wiley \& Sons, 2001.

[21] N. S. Sidney and N. J. Castellan Jr, Nonparametric Statistics for The Behavioral Sciences, $2^{\text {nd }}$ Edition, McGraw-Hill, 1988.

[22] H. Tao, P. Pinson and S. Fan, Global energy forecasting competition 2012, International Journal of Forecasting, vol. 30, no. 2, pp. 357-363, 2014

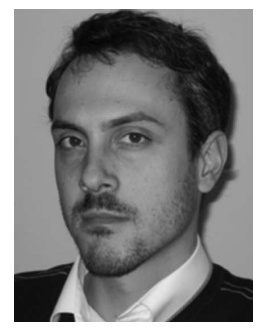

Mauro Tucci received the Ph.D.degree in applied electromagnetism from the University of Pisa, Pisa, Italy, in 2008. Currently, he is an Associate Professor with the Department of Energy, Systems, Territory and Constructions Engineering, University of Pisa. His research interests include computational intelligence and big data analysis, with applications in electromagnetism, non destructive testing, powerline communications, and smart grids.

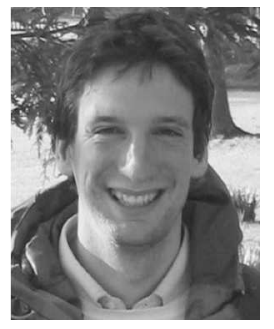

Emanuele Crisostomi received the B.S. degree in computer science engineering, the M.S. degree in automatic control, and the Ph.D. degree in automatics, robotics, and bioengineering, from the University of Pisa, Italy, in 2002, 2005, and 2009, respectively. He is currently an Assistant Professor of electrical engineering with the Department of Energy, Systems, Territory and Constructions Engineering, University of Pisa. His research interests include control and optimisation of large-scale systems, with applications to smart grids and green mobility networks.

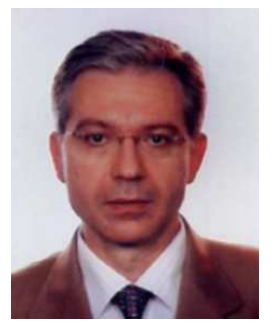

Giuseppe Giunta received the M.S. degree in physics from the University of Trento, Trento, Italy in 1985. Material science researcher at Institute of Technology and Science of Trento in 1986. Visiting researcher at Fraunhofer Institute of Non-Destructive Testing, Saarbrucken, Germany in 1991. Currently, he is Senior Professional within Eni S.p.A, Development Operations and Technology, Transmission Lines System Engineering Department for asset integrity, smart energy, technology planning and deployment. He joined Eni Group on 1987 as researcher and then entrusted in Refining \& Marketing, Gas \& Power, Midstream and Upstream division till now. Co-author of more than 90 papers published in international journals and conferences proceedings, and 13 patents. Dr. Giunta is member of the ASME, ASNT and AOGS society.

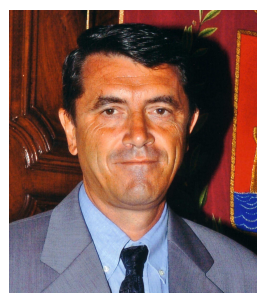

Marco Raugi received the Ph.D. degree in electrical engineering from the University of Pisa, Pisa, Italy, in 1990. Currently, he is a Full Professor of Electrical Engineering with the Department of Energy, Systems, Territory and Constructions Engineering, University of Pisa. He is the author of many papers in international journals and conference proceedings. His research interests include numerical electromagnetics, with main applications in nondestructive testing, electromagnetic compatibility, communications, and computational intelligence. Prof. Raugi was the General Chairman of the international conferences Progress in Electromagnetic Research Symposium in 2004 and IEEE International Symposium on Power Line Communications in 2007. He was the recipient of the IEEE Industry Application Society 2002 Melcher Prize Paper Award. 